\title{
A Case Report of Hide-Line-Suture-Prosthesis Repair for Anal Fistula
}

\author{
Liwen Wang1*, Yibing Li², Zhenzhen Xue'1, Qikai Liu² \\ ${ }^{1}$ Shaanxi University of Chinese Medicine, Xianyang 712046, Shaanxi Province, China \\ ${ }^{2}$ Shenzhen TCM Anorectal Hospital, Shenzhen 518031, Guangdong Province, China \\ *Corresponding author: Liwen Wang, 990685606@qq.com
}

\begin{abstract}
Hide-line-suture-prosthesis repair was introduced by Yibing Li from Shenzhen TCM Anorectal Hospital. In his early years, it was used in rectovaginal fistula, in which it achieved good curative effect. As the pathology of rectovaginal fistula and anal fistula is similar, Yibing Li innovated and applied it to anal fistula cases. This is a case report of a patient with low complex anal fistula who was treated in Shenzhen TCM Anorectal Hospital after two surgeries. The purpose of this report is to share the diagnosis and treatment experience in this case, so as to provide reference for clinicians.
\end{abstract}

Keywords: Hide-line-suture-prosthesis repair; Anal fistula; Medical record

Publication date: November 2021; Online publication: November 30, 2021

\section{Introduction}

Anal fistula is a disease recorded from medical history and is one of the most common anal diseases in clinical practice ${ }^{[1]}$. One of its sequelae is the recurrence with complicated causes. First, the internal opening of anal fistula is generally located at the highest anal resting pressure. Due to the high anal resting pressure, feces, secretions, bacteria, and other substances in the intestinal cavity may enter the surrounding tissues from the internal opening, making it difficult for the fistula to heal ${ }^{[2,3]}$. Second, it is generally difficult for anal fistula to heal by itself. Clinically, surgery is the only radical cure; however, it is common that the wound is not effectively sutured during surgery, which easily leads to mucosal dead cavity, thus increasing the rate of recurrence ${ }^{[4,5]}$. Investigations have shown that the postoperative recurrence rate of anal fistula in China is $3 \%$ to $18 \%{ }^{[6]}$. Third, there is a high expression rate of pro-inflammatory factor TNF- $\alpha$ and antiinflammatory factor IL-4 in anal fistula tissues. They play an important role in the inflammatory response of anal fistula and contribute to the occurrence as well as development of anal fistula in the interaction ${ }^{[7]}$. The treatment of low complex anal fistula is mainly through surgery, where incision is made and seton is placed in the early stage, and conventional suture is used. Postoperative wound recovery is mostly achieved by using antibiotics and patients' self-conditioning ${ }^{[8]}$. Some scholars believe that opening the mucosal dead cavity to allow it to grow in the state of normal flora is conducive to healing ${ }^{[9-10]}$.

\section{Case report}

On July 30, 2020, the resection of low complex anal fistula combined with hide-line-suture-prosthesis repair was performed. The surgical procedure was as follows: the patient was placed in the lithotomy position; after successful anesthesia, routine disinfection, towel laying, and disinfection of the lower rectum and anal canal were done. The intraoperative findings were as follows: the main fistula was located at 1 point of lithotomy position; it was about $5 \mathrm{~cm}$ long, $2.5 \mathrm{~cm}$ away from the anus, and bifurcated subcutaneously 
downward at 3 point, running about $2 \mathrm{~cm}$ long; anal sinus indentation was obvious at 6 point, and the rest was roughly the same as before; the probe was placed through the external fistula, pointing to the anal canal at 1 point; the probe smoothly protruded out from the vicinity of the tooth line at 1 point; a finger was inserted into the anal canal to extract the probe; the skin, subcutaneous tissue, and anal fistula tissue were cut with an electric knife along the probe, and the fistula opening was cut to form a new wound surface; the absorbable line of the large round needle was inserted perpendicular to the wound surface along the outer edge of the mouth and passed through the whole incision in a ring; the needle was drawn vertically from the relative position of the other end of the incision, and the absorbable line was tightened so that both ends of the wound of the inner mouth were fully fitted and the stitches were ligated; the fistula wall and connective tissue were completely removed, the sphincter was preserved, and the fistula was removed and sent for pathological examination; a drainage incision was created beside the ligation site, and an external tube was placed for drainage; at $1 / 3$ of the incision at 1 point, a large round needle with $0.5 \mathrm{~cm}$ absorbable line was inserted perpendicular to the wound surface away from the wound edge, and the wound surface was encircled in an annular way; the depth of the wound was appropriate for the sensation of the tying line; the needle was drawn vertically from the relative position of the other end of the fistula wound, and the absorbable thread was tightened to make the two ends of the fistula wound fit fully; after that, the suture line was ligated, and the fistula was sutured discontinuously with the above method, with a spacing of about $2 \mathrm{~cm}$; there was obvious induration of the anal sinus at 6 point upon exploration, and incision and drainage were performed to consider the possibility of recessive internal orifice; no active bleeding was detected in the wound; the anal canal was disinfected with $0.1 \%$ povidone iodine solution; $4 \mathrm{ml}$ of $0.25 \%$ lidocaine and $1 \mathrm{ml}$ of methylthioninium chloride was injected subcutaneously in the wound; the wound was checked again to determine if there was any bleeding; the surgical wound was wrapped and fixed with gelatin sponge and tower gauze. The surgery proceeded smoothly; intraoperative bleeding was about $6 \mathrm{ml}$, there was no blood transfusion, and $500 \mathrm{ml}$ infusion was given. The intraoperative anesthesia was satisfactory, the patient's vital signs were stable, the patient was awake after the operation, and returned to the ward safely. The specimen resected was presented to the patient, and then sent for pathological examination. The pathological tissue was visible to the naked eye: a piece of gray-red tissue of about $0.8 \mathrm{~cm} \times 0.5 \mathrm{~cm} \times 0.5$ $\mathrm{cm}$ in size. After the surgery, cefuroxime sodium $(1.5 \mathrm{~g})$ and metronidazole $(1.5 \mathrm{~g})$ were given intravenously twice a day for 3 days. During hospitalization, the dressing was changed twice a day and fumigated with traditional Chinese medicine once. The dressings were changed in time, the wound was kept clean, the drainage was unobstructed, and the drainage tube was removed after 5 to 7 days. The wound healed well after the surgery, and the patient was discharged on August 11, 2020. Upon discharge, the patient was conscious, psychologically well, able to urinate normally, no bleeding noted, but had slight pain and discomfort at the anal incision site during defecation. Anoscopy revealed that the mucosa and the incision at the tooth line were growing well.

\section{Discussion}

After the mid-20th century, even with the rapid development of surgery and the progress of surgical technology, it has been found that perioperative infection is still one of the main reasons for surgical failure ${ }^{[10]}$. As understood, the treatment goal of anal fistula is to accurately find the internal orifice, reduce the resting pressure of anal canal, correctly handle the wound, and prevent its recurrence. This is the key to reduce pain ${ }^{[11]}$. Hide-line-suture-prosthesis repair is a surgical procedure under the condition of normal flora. It can prevent infection by hiding the suture stroke, reducing the suture level, opening the mucosal dead cavity, establishing drainage wound at the ligation, and avoiding deep infection along the suture 
pinhole ${ }^{[10]}$. The application of hide-line-suture-prosthesis repair in patients with low complex anal fistula can shorten the surgery time, accelerate wound healing, prevent fistula recurrence, and maintain the function of anal sphincter. It has been applied in clinical practice to ensure long-term curative effect and provide a good way for the treatment and prognosis of anal fistula.

\section{Disclosure statement}

The authors declare that there is no conflict of interest.

\section{References}

[1] Zou D, Li P, 2016, Research Progress of External Chinese Medicine Treatment after Anal Fistula Operation. Information on Traditional Chinese Medicine, 33(3): 129-131.

[2] Yuan H, Pan C, Liu Z, et al., 2019, Clinical Efficacy of Two Sphincter Preserving Methods in the Treatment of Complex anal Fistula. The Journal of Practical Medicine, 35(18): 2937-2941.

[3] Corman ML, 2016, Corman Colorectal Surgery: Sixth Edition [Fu C, Wang Ji, Wang S, Trans.], Shanghai Science \& Technical Publishers, Shanghai, 1.

[4] Fer AO, 2018, Perianal Inflammatory Diseases: Classification and Imaging. Radiologe, 58(4): $344-$ 354.

[5] Jia K, 2019, Discussion on the Effect of Ligation of Intersphincteric Fistula in the Treatment of High Simple Anal Fistula. Contemporary Medicine Forum, 17(9): 73-74.

[6] Shi Y, Shi C, 2015, Observation on the Curative Effect of Sphincter Preservation in the Treatment of Complex anal Fistula. Guangzhou Medical Journal, 3(2): 24-26.

[7] Li L, Jin Y, Zhang X, et al., 2020, Expression and Significance of TNF- $\alpha$ and IL-4 in Perianal Abscess and Anal Fistula. Zhejiang Journal of Integrated Traditional Chinese and Western Medicine, 30(07): 558-561, 614.

[8] Jia X, Hu X, Lv Z, 2019, Clinical Diagnosis and Treatment of Refractory Hyperthyroidism. Chinese Journal of Practical Internal Medicine, 39(4): 316-320.

[9] Yu W, Wang Z, 2019, Analysis of the Therapeutic Effect of Interanal Sphincter Fistula Ligation for Complex Anal Fistula. Guide of China Medicine, 17(12): 103-104. DOI: 10.15912/j.cnki.gocm.2019.12.075

[10] Li Y, Xing G, Guo W, et al., 2012, Clinical Effect of Hide-line-suture-prosthesis Repair in the Treatment of Middle and Low Rectovaginal Fistula. West China Medical Journal, 27(10): 649-651.

[11] Liu LC, Zhang YR, Su Y, et al., 2019, Lidocaine combined with Compound Betamethasone Partial Impregnation for Anal Fistula Incision the Effect of Post-Pain. Journal of Clinical Rational Drug Use, 12(12): 29-31. 